\title{
Fortifications in the territory of Alessandria: an heritage to preserve and enhance
}

\section{Anna Marotta}

Politecnico di Torino - Department of Architecture and Design (DAD), Torino, Italy, anna.marotta@polito.it

\begin{abstract}
From the Middle Ages and up to the nineteenth century, the heritage of architecture for the defense in the territory of Alessandria in Piedmont constitute a coherent system of examples that weld the theory of handbooks with the practice of the construction, intended to protect places and cities in this region: Casale Monferrato, Alessandria, Gavi etc. From Sebastien Vauban to the "father Engineer" Vincenzo da Fiorenzuola and to Lorenzo Bernardino Pinto, Antonio Ignazio Bertola, Gaspare Beretta (and beyond) the presence of designs and achievements in this area is configured as authentic and tangible "anthology of models for the defense "in Europe, for which the writer has in place for twenty years a systematic scientific research. On this occasion, we will address the possibility of rebuilding, also virtually, those episodes in the territory as a whole, to arrive at a deeper understanding both for the disclosure, as for cultural tourism.
\end{abstract}

Keywords: representation, treatise and handbooks, architectural heritage, virtual reconstruction.

\section{Premessa}

La ricerca considera temi locali del territorio dell'Alessandrino e li riporta ad una dimensione di più ampio respiro considerando l'ambito nazionale e internazionale sfruttando punti di vista e di analisi che coinvolgono la dimensione interdisciplinare caratteristica di ogni progetto sviluppato ad ampio raggio. La Cittadella di Alessandria in Piemonte è oggetto di numerose petizioni e iniziative per possibili opzioni di candidatura tra i beni tutelati dall'UNESCO. La fortezza, vera e propria antologia tipologica "nel territorio della "difesa" europeo, è programmaticamente e sistematicamente inserita nella "rete" dei sistemi difensivi europei, mediante appropriate mappature e periodizzazioni. Gli studi ventennali condotti da Anna Marotta sul tema delle fortificazioni dell'Alessandrino (con la collana edita dalla Fondazione Cassa di Risparmio di Alessandria) sono stati sviluppati ulteriormente dal 2010 in collaborazione con Serena Abello in occasione delle ricerche legate al dottorato di ricerca in Beni Culturali (Politecnico di Torino) conclusesi con la tesi "Dalla tradizione all'innovazione: la virtualità a supporto di memoria e conoscenza.

La Cittadella di Alessandria". I risultati raggiunti sono stati poi convogliati all'interno di nuovi filoni di ricerca progettati da Anna Marotta in relazione a una nuova possibile destinazione proposta per la Cittadella di Alessandria come luogo parte di possibili percorsi dello spirito "dalla guerra alla pace", concetto che potrebbe estendersi a un più ampio sistema di fortezze. A tal proposito è stato coinvolto anche l'ICOMOS Italia che potrà svolgere un ruolo strategico per perseguire in modo più efficace questi obbiettivi. 


\subsection{La Cittadella di Alessandria nella rete dei luoghi della difesa}

La conservazione del patrimonio culturale anche del paesaggio - nel suo insieme e (anche) nella sua complessità consente di valorizzarlo meglio e più consapevolmente.

Questo vale anche per la Cittadella di Alessandria in Piemonte, che è stata oggetto di petizioni e iniziative anche per una possibile candidatura UNESCO.

La Cittadella di Alessandria in Piemonte (1738), vera e propria antologia tipologica "nel territorio europeo di difesa", è stata programmaticamente e sistematicamente inclusa nella "rete" dei sistemi di difesa in Europa a luogo e ampiamente studiati da Anna Marotta, attraverso la mappatura e la periodizzazione del caso. La nuova proposta è una Cittadella di Alessandria "dalla guerra alla pace", un concetto che potrebbe essere diffuso a un più ampio sistema di fortezze. A tal fine, l'ICOMOS Italia attraverso il suo presidente, Maurizio Di Stefano - svolgerà un ruolo strategico nel modo più efficace per perseguire questi obiettivi.

Si tratta di una fortezza, una grande macchina da guerra, ma la proposta di Marotta intende trasformarla in un "luogo-museo" per il culto, la tolleranza e la pace attraverso la creazione di un "percorso per lo spirito". Ciò significa che per aggiornare e invertire il concetto dei Sacri Monti tra Riforma e Controriforma: i limiti non sono più territoriali o concettuali, ma $\mathrm{i}$ rapporti tra $\mathrm{i}$ luoghi diventano dinamici attraverso strumenti di scambio tra culture e fedi, in materia di "identità in differenze". Potrebbe essere l'applicazione pratica di ciò che Gandhi ha detto: "Forse ci vorrà del tempo prima che la legge dell'amore venga presa in considerazione negli affari internazionali, ma se vogliamo salvarci e dare un importante contributo al progresso del mondo, dobbiamo seguire con convinzione e diligenza la via della pace".

La lettura della fortezza alessandrina può essere ricondotta all'interno di molteplici sistemi di osservazione.

Tra le ricerche coordinate da Anna Marotta presso il Dipartimento di Architettura e Design del Politecnico di Torino, Serena Abello ha approfondito, con la sua tesi di dottorato, alcune tematiche legate alla proposta di alcuni possibili scenari per delineare - in termini congruenti e rispettosi del luogo - un progetto di comunicazione che consenta una lettura integrata della fortezza in sistema più ampiamente inteso.

Le vicende della Cittadella di Alessandria sono concatenate con quelle di numerose fortezze del territorio circostante ma anche - e soprattutto del territorio regionale, nazionale e internazionale.

Essa può essere concepita come luogo di scambio culturale che - coinvolgendo Amministrazioni, enti pubblici e privati - possa stimolare la proposta per la discussione e il dibattito attraverso l'organizzazione di occasioni di incontro come convegni - inerenti l'ambito locale, nazionale ma, soprattutto internazionale favorendo l'opportunità della prestigiosa location - sede di tali iniziative - di essere conosciuta e apprezzata.

A tal proposito Anna Marotta ha considerato la possibilità di inserire questo sito nella "rete" dei luoghi un tempo destinati alla "guerra" ed oggi ipotetici centri di incontro e "di pace".

Dalle suggestioni originate mediante tentativi di comparazione (analizzati nella pubblicistica edita degli ultimi vent'anni e più), sono scaturite ricerche inerenti le similitudini esistenti tra una serie di esempi prescelti attraverso criteri (caratteri visivi, geometrici e strutturali) che hanno consentito il paragone di esempi coevi alla Cittadella di Alessandria (locali, nazionali e internazionali). Tale selezione, è stata eseguita in riferimento alle fonti consultate: alcuni sono luoghi menzionati nella pubblicistica di riferimento come, ad esempio, nel volume dedicato alla fortezza alessandrina curato proprio da Anna Marotta. Altri parametri di selezione hanno condotto a una casistica di fortezze appartenenti all'opera di Vauban alcune delle quali sono parte del patrimonio riconosciuto dall'UNESCO - mentre più in generale sono stati individuati i sistemi di difesa realizzati "alla moderna". 

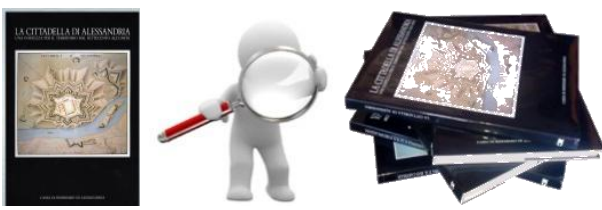

Fig. 1- Anna MAROTTA ha curato nell'ultimo ventennio una collana dal titolo "Sistemi e fortificazioni nell'Alessandrino" per la Cassa di Risparmio di Alessandria.

La selezione critica degli esempi di Cittadelle e Città di fondazione esistenti in ambito europeo delinea un percorso conoscitivo e cognitivo su similitudini e/o difformità tra le diverse realtà e tipologie.
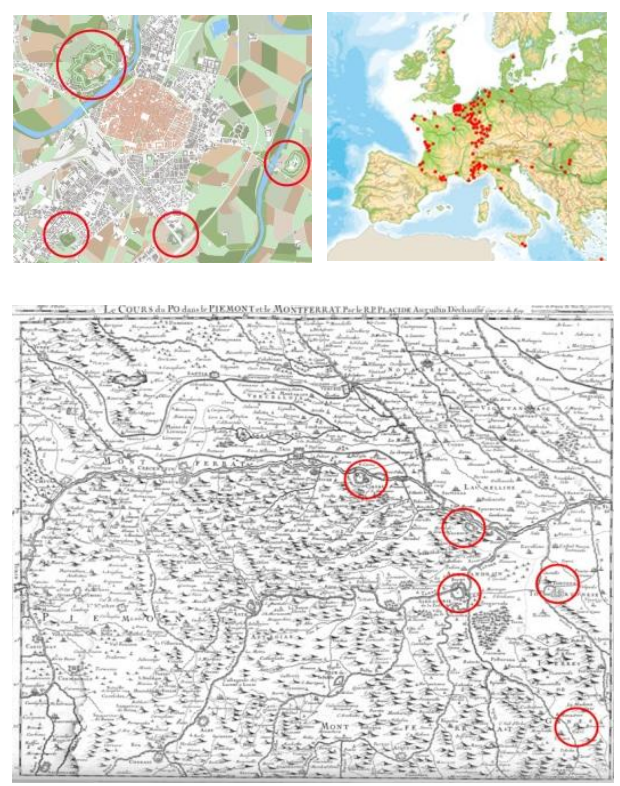

Fig. 2- Nel teatro europeo i luoghi della difesa risultano polarizzati in una reta ampia e diramata

Attraverso il confronto del territorio della difesa nell'Alessandrino (visto come ampia antologia di tipi di fortificazioni e di architettura militare) con le analoghe realtà europee, propone una visione sistematica di fortificazioni, allargata ad alcune città di fondazione. Ne risulta un'Europa costellata di esempiLe categorie individuate per la classificazione e la catalogazione degli esempi selezionati prevedono di osservare alcune caratteristiche generali riportate su una specifica scheda di analisi.
In particolare, l'ipotesi di Serena Abello delle schede iconografiche, ipotizza (2013):

-fonti documentarie;

-localizzazione della fortezza;

-periodo di riferimento;

-autore dell'opera;

-tipologia del territorio;

-tipologia del sistema di difesa;

-funzione originaria;

-forma;

-numero dei lati del poligono;

-stato di conservazione del segno sul territorio;

-legami con la Cittadella di Alessandria.

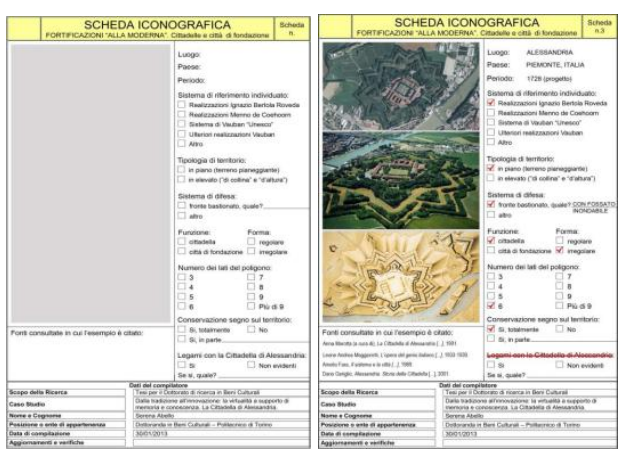

Fig. 3- Schede iconografiche. Fortificazioni alla moderna, Cittadelle e Città di fondazione. Elaborate da Serena Abello, 2013. (www.marilin.eu). A destra alcuni esempi

La casistica definita consente di analizzare il rapporto tra il "disegno" delle fortificazioni e il "disegno" della città: il valore di vivo segno visibile, la chiarezza dell'impianto del sistema, la canonicità dell'applicazione delle regole confermano la Cittadella di Alessandria come modello presente in tutta la trattatistica militare più attenta e importante a livello europeo.

Seguendo le direttive metodologiche usate nelle esperienze di studio di Anna Marotta si è deciso di osservare una selezione di casi rispetto alla sua conformazione fisica, passata e presente, con l'obiettivo di rileggere il passato nei segni della realtà attuale. Questo approccio ha permesso di registrare come - nelle diverse realtà, con motivazioni molto dissimili tra loro - le strutture per la difesa siano transitate nel tempo. Si tratta di strutture con forme geometriche differenti (che, a volte rispettano fedelmente le leggi 
teoriche, altre seguono le esigenze specifiche del luogo) disegnano e rimodellano il territorio: dal castello alla cittadella, fino alla definizione delle città di fondazione, sono ripercorse e perseguite le regole della geometria.
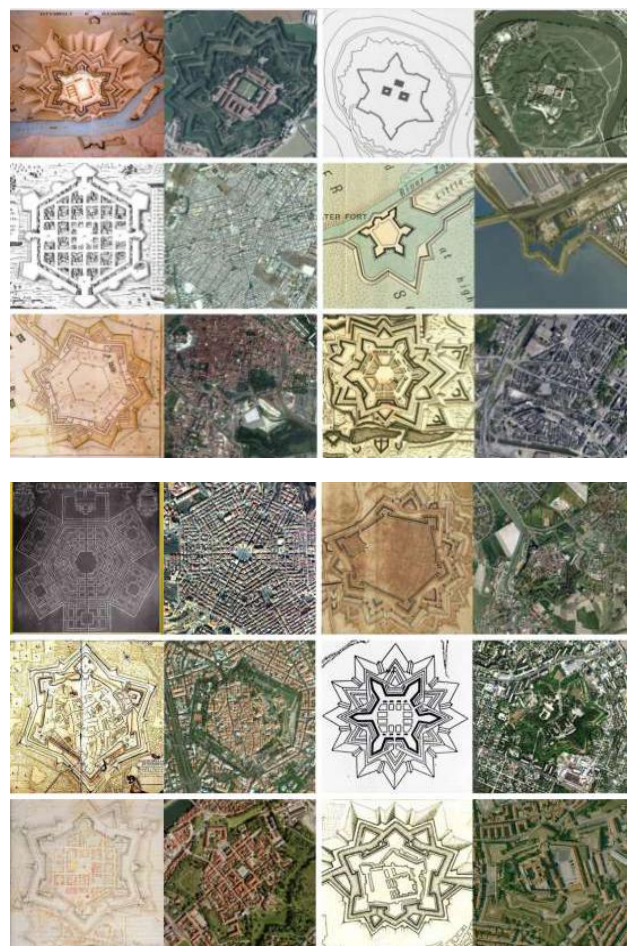

Fig. 4- Selezione di casi studio rispetto al criterio della geometria della forma esagonale regolare e irregolare. Una possibile rete di fortezze "dalla guerra alla pace". Elaborazione di Serena Abello 2013.

Il quadro sinottico descrive una selezione definendo come criterio quello della geometria regolare e irregolare della forma esagonale - del primo accorpamento preliminare per la catalogazione delle informazioni inerenti le fortificazioni. Essa è stata consentita a seguito dei dati raccolti nelle Schede iconografiche. Fortificazioni "alla moderna". Cittadelle e città di fondazione. Insieme al caso alessandrino possono essere comparati casi quali Charleroi in Belgio, Karlovac in Croazia, Naarden nei Paesi Bassi, Perpignan e Charleville in Francia, ecc.

\subsection{Itinerari dello spirito nei luoghi della difesa}

Tra i possibili risultati relativi ai temi dei "Percorsi dello Spirito", è stato possibile introdurre un altro progetto, che unisce diversi campi di ricerca, in cui l'autore e il suo gruppo di ricerca sono impegnati da lungo tempo.

Il nostro Papa Bergoglio porta lo stesso nome del quartiere che oggi ospita la Cittadella di Alessandria. A questo proposito, il secondo progetto riguarda l'importante complesso fortificato del Piemonte (oggi libero dall'occupazione militare) in una proposta, che intende convertire un luogo di guerra in un luogo di pace: una sorta di museo, dedicato a multifede, tolleranza e la spiritualità, attraverso la realizzazione di un percorso dello Spirito, non diverso da quello concepito per l'Ospedale. Si basa quindi su alcuni principi fondamentali: informazione, conoscenza, comprensione e condivisione.

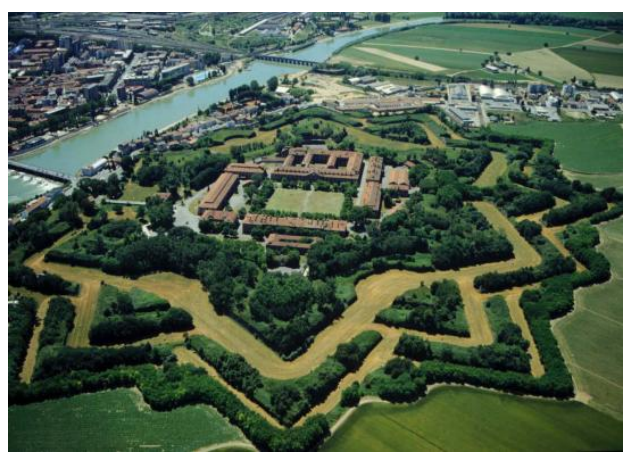

Fig. 5- Vista aerea della Cittadella di Alessandria

All'interno di più ampie e complesse iniziative per la riorganizzazione e la rifunzionalizzazione delle strutture ospedaliere della città di Torino e in particolare della cosiddetta "Città della Salute e della Scienza", in data 04/02/2013 veniva sottoscritto il "Contratto di ricerca tra l'Agenzia Regionale per i Servizi Sanitari (AReSS) e il Dipartimento di Architettura e Design (DAD) del Politecnico di Torino relativo allo sviluppo del progetto "Stanze del silenzio/Percorsi dello Spirito". Responsabile scientifico la prof. Anna Marotta (DAD). 
Il progetto "Stanze del Silenzio" si inserisce nei percorsi progettuali di A.Re.S.S. quale declinazione tematica dei criteri e indirizzi generali di pianificazione e progettazione delineati nel Master Plan della Città della Salute e della Scienza di Torino. In particolare si qualifica come approfondimento specifico dei temi relativi al rapporto ed alle modalità di interazione tra "funzioni e luoghi di cura" e "funzioni e luoghi di culto" in riferimento a un contesto ospedaliero caratterizzato sia da una grande complessità di prestazioni e relazioni, sia da un'elevata eterogeneità dell'utenza sotto gli aspetti anagrafici, sociali e culturali.

Accanto ai luoghi di culto cattolico sono infatti da valutarsi le possibilità di realizzazione di sale del silenzio o di luoghi "multiculto", ovvero la realizzazione di luoghi dedicati a culti specifici. In questo senso i luoghi dedicati al culto dovranno tener conto delle diverse esigenze e connotazioni dell'utenza a cui sono rivolti e dovranno trovare idonea localizzazione rispetto alle funzioni ed alle attività svolte in Città della Salute e della Scienza di Torino.

All'interno del presente lavoro, si riconferma (per l'intero sistema), anche alla luce di quanto finora espresso, il concetto e il valore di "percorso della mente e dell'anima": non solo per convogliare in un luogo preciso chi abbia esigenze di raccoglimento, riflessione e meditazione, ma anche per

\section{"irradiare spiritualità"}

in tutto il complesso di Città della Salute e della Scienza di Torino.

Si rafforza così il concetto di "sostenibilità dello spirito": una sostenibilità non solo ecologica e/o tecnologica, ma anche etica e culturale, secondo la quale la dimensione del pensiero e dell'anima costituisce un'esperienza forte (costante e condivisa, non relegata a pochi momenti della giornata o della settimana). Una dimensione che ci aiuti ad accettare le personali difficoltà del vivere, per aggiungere qualità non solo materiali alla nostra esistenza, ma anche per trovare valori comuni nel confronto collettivo, nel rispetto del complesso sistema delle differenze religiose: per "fare comunità". Un'educazione alla condivisione, dunque, articolata secondo un percorso che potrebbe essere suddiviso nelle seguenti fasi:

\section{informare, conoscere, comprendere, condividere}

Tra i possibili risultati relativi ai temi dei "Percorsi dello Spirito", è stato possibile introdurre un altro progetto, che unisce diversi campi di ricerca, in cui l'autore e il suo gruppo di ricerca sono impegnati da lungo tempo.

Alcune proposte progettuali prevedono l'inserimento del punto informativo (in base, ad esempio, di calendari diversi Festa), simbologia di varie religioni, templi e case di preghiera, grazie alla realizzazione di proiezioni digitali interattivi, che potrebbero coinvolgere gli utenti nelle esperienze multiculturali, con grande condivisione affettiva.

Tra i primi riferimenti, potremmo ricordare il Centro Nobel per la Pace, che ha sede in una ex stazione ferroviaria di Oslo, ospiterà il Campo Nobel, una suggestiva installazione di arte, che simboleggia un giardino.

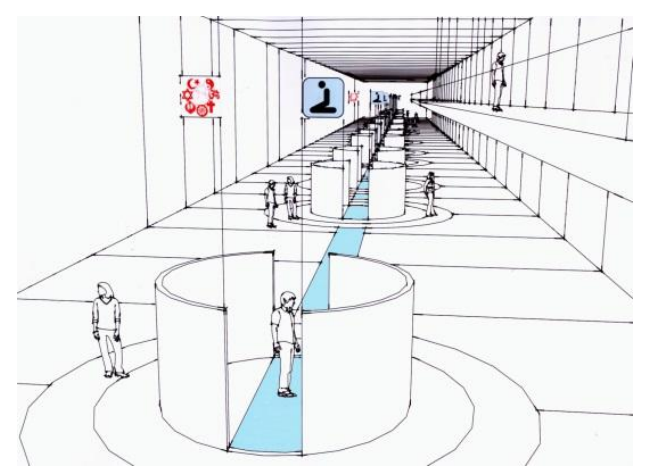

Fig. 6- Simulazione meta progetto - possibili suggestioni formali e funzionali - elaborazione a cura del Politecnico di Torino-DAD

Un altro esempio - che non può essere dimenticato - è il Centro per la pace (Peres Peace House) di Jaffa, in Israele commissionato da Simon Peres (Premio Nobel per la Pace), il cui progettista è Massimiliano Fuksas.

Il centro è stato concepito come un luogo destinato a diventare la sede principale di discussione delle iniziative arabo-israeliana, 
nella prospettiva di un graduale processo di pacificazione in Medio Oriente, la promozione di progetti per l'emancipazione dei gruppi locali e dei singoli individui. Il primo evento che si è svolto all'interno dell'edificio è stata la cerimonia per la laurea di ventuno donne, la maggior parte di loro erano arabe.
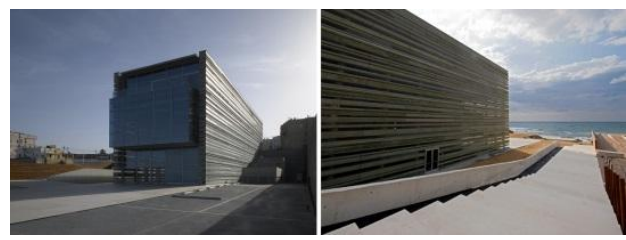

Fig. 7- Centro per la Pace (Peres Peace House), Jaffa, Israele. Progetto di Massimiliano Fuksas (2009). Immagine tratte da: (a sinistra) http://www.arthitectural.com/massimiliano-anddoriana-fuksas-peres-peace-

house/peres_peace_house_019/ e (a destra) http://www.dailytonic.com/peres-peace-housejaffaisrael/

Il Centro per la Pace è un parallelepipedo ottenuto dalla stratificazione di piani irregolari di calcestruzzo e vetro traslucido. La struttura è illuminata durante tutto il giorno e tutta la notte, anche grazie al progetto di illuminazione interna, offrendo un'immagine quasi magica, che convogli il messaggio spirituale espresso dall'architettura.

In conclusione, se l'architettura può essere "terapeutica", può essere compresa come bene culturale e può (deve) essere un luogo per costruire la pace; la pace dell'anima, e non solo individuale. Una proposta è perciò quella degli Itinerari e Percorsi dello Spirito, che - per il momento - sono stati proposti nei luoghi ospedalieri, ma che possono essere implementati in altre situazioni che coinvolgono la collettività. Possiamo essere d'accordo con Gandhi, quando afferma: "Forse ci vorrà del tempo, prima che la Legge dell'Amore è adottata in affari internazionali, ma se vogliamo salvare noi stessi e dare un importante contributo al progresso del mondo, dobbiamo percorrere con determinazione la via della pace".

L'edificio appoggia su un basamento monolitico, dove sono distribuiti gli ingressi per autoveicoli e pedoni.

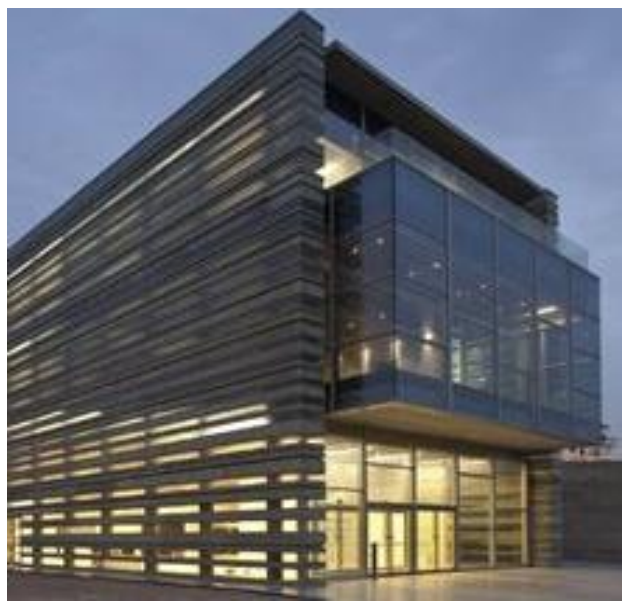

Fig. 8- Centro per la Pace (Peres Peace House), Jaffa, Israele. Progetto di Massimiliano Fuksas (2009).

\section{References}

Marotta A., Lombardi M. (2013), Anima valens in corpore aegro. Luoghi dello spirito nella Città della Salute e della Scienza di Torino. Percorsi di cura dello spirito. Sale del silenzio, sale multiculto, chiese. Ananke Editore. Roma. pp. 1-94.

Marotta A. (2013), "Cultura della visione per conservare e valorizzare: il caso della Cittadella di Alessandria / Culture of vision to preserve and enhance: the case of Citadel of Alexandria". In: $35^{\circ}$ Convegno internazionale dei docenti della Rappresentazione. Patrimoni e Siti Unesco. Memoria, misura e armonia / Heritage and Unesco Sites. Memory, measure and harmony, Matera, 24-26 ottobre 2013. pp. 615-622.

Marotta A. (2013), "Visione sostenibile (Sustainable Vision in the Conservation of Cultural Heritage Project)". In: Heritage architecture landesign focus on conseration regeneration innovation, Le vie dei Mercanti - XI Forum Internazionale di Studi, Aversa/Capri, 13-15 giugno 2013. pp. 1210-1219. 
Marotta A. (2012), "From drawing in the treatises to building in the construction sites: examples in Piemonte". In: Beetween East and West. Transposition of cultural systems and military technology of fortified landscapes, Poppi (AR) - Firenze, 7-13 maggio 2012. pp. 76-80.

Marotta A. (2012), "Geometria e costruzione: modelli mentali e tipi realizzati nel territorio della difesa”. In: disegnare con..., vol. 5 n. 9, pp. 161-166. - ISSN 1828-5961.

Marotta A., Abello S. (2012), "Paesaggi culturali in transizione: sistemi della difesa del territorio dall'Unità d' Italia all'Unione europea". In: Topscape paysage, vol.1. Overvi, pp. 896-925.

Marotta A.G. (2005), "Il disegno delle fortificazioni milanesi nell'Alessandrino". In Graziella Colmuto Zanella, Flavio Conti, Luciano Roncai (a cura di), La difesa della Lombardia spagnola. Atti del Convegno di Studi, Politecnico di Milano, 1998. pp. 279-296.

Marotta A.G.; A. G (2002), "La "figura urbana" nei disegni della città difesa. Fortezze conservate e fortezze cancellate nell'Alessandrino". In Il disegno della città. Opera aperta nel tempo. Convegno Internazionale A.E.D.

Marotta A.G. (a cura di) (1991), La cittadella di Alessandria una fortezza per il territorio dal settecento all'unità. Cassa di Risparmio di Alessandria. Alessandria.

Marotta A.G. (1990), "Casale baluardo d'Italia nella strategia risorgimentale”. In: La Cittadella di casale da fortezza del Monferrato a baluardo d'Italia 1590-1859. Cassa di Risparmio di Alessandria. Alessandria.

Marotta A.G. (1990) (a cura di), La Cittadella di casale da fortezza del Monferrato a baluardo d'Italia 1590-1859. Cassa di Risparmio di Alessandria. Alessandria. 
